\title{
Enquête
}

Archives de la revue Enquête

5 | 1997

Débats et controverses

\section{Le questionnement logiciste et les conflits d'interprétation}

Logicist Questioning and Conflicts of Interpretation

Jean-Claude Gardin

\section{(2) OpenEdition}

1 Journals

Édition électronique

URL : http://journals.openedition.org/enquete/1043

DOI : 10.4000/enquete.1043

ISSN : 1953-809X

Éditeur :

Cercom, Éditions Parenthèses

Édition imprimée

Date de publication : 1 septembre 1997

Pagination : 35-54

Référence électronique

Jean-Claude Gardin, « Le questionnement logiciste et les conflits d'interprétation », Enquête [En ligne], 5 | 1997, mis en ligne le 15 juillet 2013, consulté le 21 mars 2020. URL : http://

journals.openedition.org/enquete/1043; DOI : https://doi.org/10.4000/enquete.1043

Ce document a été généré automatiquement le 21 mars 2020. 


\title{
Le questionnement logiciste et les conflits d'interprétation
}

\author{
Logicist Questioning and Conflicts of Interpretation
}

\author{
Jean-Claude Gardin
}

1 La première controverse dont je dois faire état est celle, courtoise mais que je croyais ferme, qui m'opposa d'abord à l'éditeur de ce numéro d'Enquête lorsqu'il eut l'amabilité de m'inviter à y collaborer. À quoi devais-je cette offre? Sans doute à la place qu'occupe l'analyse des conflits d'interprétation dans le programme logiciste auquel on m'associe volontiers ${ }^{1}$. Conflits, débats, controverses, nous sommes bien, en effet, dans un même paysage ; toutefois, je pensais avoir déjà trop écrit sur la façon dont l'analyse logiciste des constructions interprétatives s'y déploie pour qu'il fût utile, une fois encore, de «reprendre la question ». À mes réticences des objections furent invoquées, qui me persuadèrent de tenter un nouvel essai ; mais essayer quoi ? Avancer des idées neuves sur le sujet? J'avoue n'en avoir acquis aucune depuis des années, comme en témoigne l'abondance des redites dans mes écrits. Commenter les idées neuves d'autrui en ces mêmes matières? L'obstacle, ici, est que je suis plus sensible à la diversité des variations qu'à l'originalité des thèmes dans la marée toujours montante des livres consacrés aux questions d'épistémologie dans les sciences humaines, à quelque enseigne qu'on les place - histoire des idées, sociologie de la connaissance, philosophie des sciences, etc. C'est donc à une tâche plus modeste que je m'essaierai dans le cas présent : je tenterai de résumer les raisons qui me paraissent assurer la pérennité du questionnement logiciste, au-delà des façons toujours plus nombreuses et toujours plus savantes de l'éluder. Ce parti m'oblige à rappeler quelques positions du programme logiciste pour les besoins de la démonstration.

\section{Rétrospective}

2 [1] Un premier point concerne la différence entre l'étude socio-historique des controverses et l'analyse logiciste des conflits d'interprétation. L'une vise à dégager les facteurs de toute espèce qui sont à l'origine de «divergences de vue » prises dans le 
sens le plus large et dans les contextes les plus variés; l'autre considère les phénomènes de multi-interprétation dans le champ d'une discipline, ou mieux d'une problématique scientifique particulière, pour mettre en lumière les sources techniques des divergences constatées (le terme «techniques» s'entend ici des techniques de la construction savante, ramenée à ses composantes symboliques essentielles).

3 Un exemple, s'il en faut, pris dans ma propre discipline, l'archéologie: le cas de Stonehenge. On sait la variété des interprétations auxquelles a donné lieu le cercle de monolithes qui a rendu le nom de cette localité célèbre parmi les préhistoriens du monde entier. Un livre est paru qui défend une thèse nouvelle touchant l'origine du monument: il serait l'œuvre d'une communauté bretonne, exogène par rapport au peuplement connu du Wiltshire à l'époque de Stonehenge. Des voix se sont immédiatement élevées outre-Manche pour contester cette dépossession hypothétique; une controverse s'est ainsi engagée, qui ne manquera pas d'intéresser à juste titre la sociologie de la préhistoire. À terme, toutefois, le débat sera tranché par des critères scientifiques qui l'emporteront sur tout autre "facteur » socio-historique de la controverse. C'est au repérage de ces critères-là de décision que s'attache principalement l'analyse logiciste des raisonnements.

4 [2] Une différence connexe sépare cette entreprise des études consacrées aux processus de découverte. La schématisation d'une construction savante, dans le premier cas, ne prétend pas retracer les cheminements intellectuels qui ont conduit à sa formulation finale dans une publication scientifique. Soyons plus précis : les opérations mobilisées dans un texte scientifique pour passer de la base de données $\{\mathrm{P} o\}$ aux hypothèses ou conclusions $\{\mathrm{Pn}\}$ de l'auteur - ou l'inverse - n'ont a priori rien à nous dire quant aux mécanismes psychologiques mis en jeu dans l'émergence de $\{\mathrm{Pn}\}$ ou dans la recherche de sa démonstration sur la base de $\{$ Po\}. (Cf. schéma). 


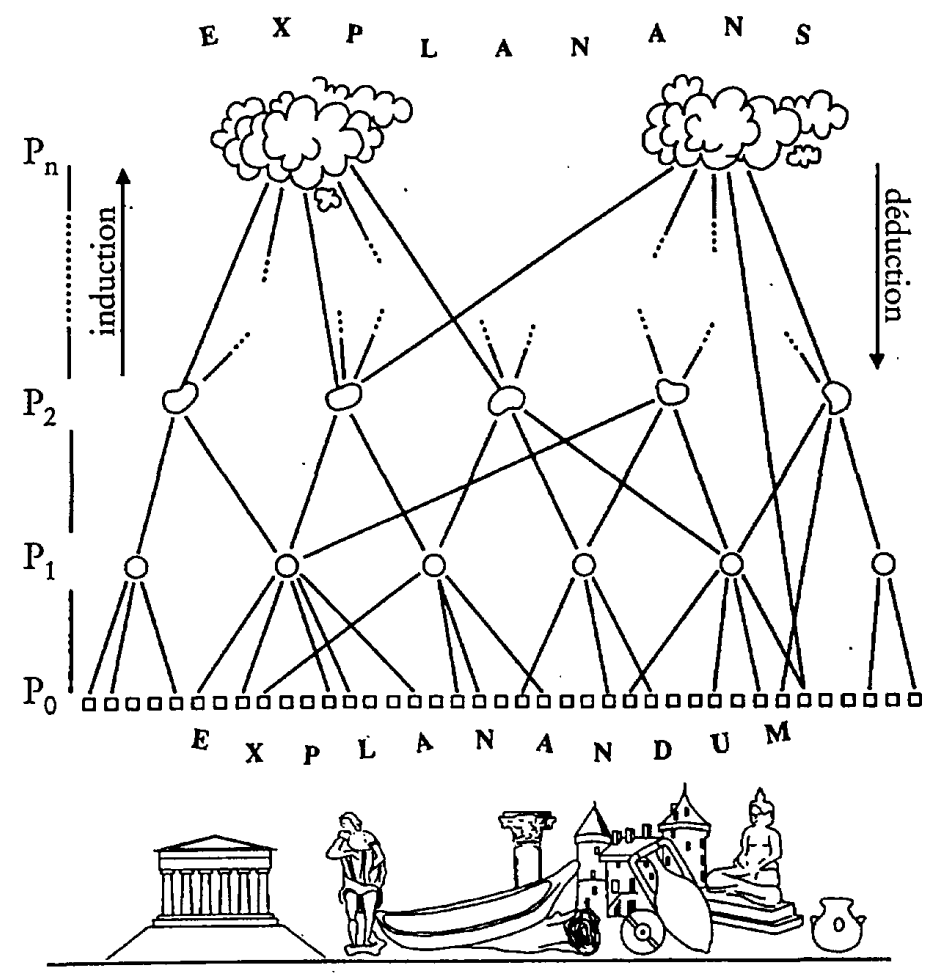

Les deux composantes des constructions savantes dans les schématisations logicistes a - la base de données $\{\mathrm{P} O\}$ (composante horizontale, en extension);

$\mathrm{b}$ - les opérations de réécriture $\{\mathrm{Pi}\} \rightarrow\{\mathrm{Pi} \pm 1\}$ (composante verticale, en intension).

Reproduit d'après J.-C. Gardin, Une archéologie théorique, Paris, Hachette, 1979, p. 177, fig. 23.

5 [3] L'étroitesse du cadre méthodologique ainsi tracé et la relative pauvreté des ambitions qu'on y poursuit sont cause de la façon terre à terre dont sont ici définies les controverses. On les considère à deux échelles : a) une échelle macroscopique lorsqu'on se borne à constater qu'à partir d'observations empiriques et de présuppositions théoriques plus ou moins comparables (niveau Po), des auteurs divers fondent des hypothèses ou des conclusions différentes (niveau Pn); b) une échelle microscopique lorsque le même phénomène se manifeste au niveau des opérations successives de réécriture $\{\mathrm{Pi}\} \rightarrow\{\mathrm{Pi} \pm 1\}$ qui relient Po à Pn (ou l'inverse), les mêmes antécédents donnant lieu à des dérivations différentes selon les auteurs ou selon les moments du raisonnement.

6 Le repérage des divergences de type a) est relativement facile dans un domaine ou sur un thème de recherche précis; il invite à explorer les divergences de type b), les plus intéressantes au regard d'une épistémologie pratique. La détection et la schématisation des controverses de cet ordre constituent la part la plus ardue du programme logiciste ; c'est aussi celle qui, selon moi, le justifie.

7 [4] A quoi servent, en effet, de tels repérages? La règle du jeu convenue dans ce programme est que les divergences ainsi compilées méritent a priori d'être traitées comme des problèmes justiciables peut-être de quelque solution, et exigent par conséquent qu'on s'emploie à chercher celle-ci par les voies habituelles de la recherche dans les disciplines empiriques ${ }^{2}$. L'aboutissement est, au mieux, une contextualisation des opérations d'inférence $\mathrm{p} \rightarrow \mathrm{q}$, lorsqu'on parvient à caractériser le domaine d'une règle équivalente $\mathrm{p}^{\prime} \rightarrow \mathrm{q}$ où $\mathrm{p}^{\prime}$ contient des spécifications non formulées dans $\mathrm{p}$. L'archéologie, pour m'en tenir à une discipline que je connais, regorge d'exemples 
parfaitement réussis dans ce sens; et le progrès des connaissances est là manifeste, dans l'affinement continu de telles règles substituées à des pratiques discursives plus lâches ou plus rhétoriques. Il serait surprenant que le phénomène n'eût aucun parallèle ailleurs, comme le voudrait la thèse du caractère non cumulatif des résultats de la recherche dans les sciences de l'homme.

8 [5] Il reste que l'exercice précédent a ses limites: un très grand nombre de nos pratiques argumentatives résistent à ces tentatives de régulation, au moins si l'on écarte cette forme spécieuse de rationalisation qui consiste à inclure dans p' le nom de l'auteur de l'opération $\mathrm{p} \rightarrow \mathrm{q}$... On aurait tort cependant de ne voir là qu'un truc, et plus encore de le railler; car il est facile de démontrer que l'apprentissage académique de ce qu'on désigne et pratique dès l'école sous le nom d'«explication de texte » passe largement par l'acceptation de cette rationalité-là'.

Le questionnement logiciste s'étend au-delà des limites que je viens d'évoquer : à quelle logique, s'il en est, obéissent nos pratiques interprétatives lorsque les opérations qui les constituent ne se prêtent pas au processus de régulation rappelé plus haut ? La réponse que je proposai d'abord fut, plutôt qu'une logique, un art de l'expression qui captive l'intérêt du lecteur et donne à l'interprétation un pouvoir de persuasion comparable à celui des récits de fiction lorsqu'ils ont cette qualité - une qualité littéraire, par conséquent, qui n'a pas peur de dire son nom, ni d'affirmer qu'elle n'est pas celle de n'importe quel « récit ${ }^{4}$ ».

[6] Cette dualité Science/Littérature n'avait assurément rien d'original : j'ai naguère tenu à rappeler que trente ans avant C. P. Snow un philosophe oublié du nom de Pius Servien l'avait admirablement assise à travers une illustration des deux chemins de la connaissance qu'ouvraient, selon ses termes, le langage des sciences et le langage lyrique, sans laisser aucune place à un troisième ${ }^{5}$. Par la suite, le langage lyrique a été revendiqué par des historiens rebelles au langage des sciences, mais sous couvert d'un qualificatif « narratif » moins exigeant que le lyrique ou le littéraire 6 .

11 Un glissement s'est néanmoins produit, de Pius Servien (ou Paul Valéry, qui l'approuvait) à nos contemporains. Pour les premiers, les deux voies de la connaissance étaient alternatives, ou mieux complémentaires, au service de la pénétration de toutes choses, naturelles ou humaines ; pour les seconds, le mode narratif semble plutôt conçu comme un substitut du mode logico-scientifique, jugé impropre à l'interprétation des faits humains. L'embarras de ce dernier parti, à mes yeux, est que la narrativité est un trait caractéristique de toutes sortes d'écrits - contes, reportages, romans, mémoires, et jusqu'aux racontars les plus anodins de la vie quotidienne - de sorte qu'il appartient au chercheur de distinguer les siens d'une manière plus précise afin de sauvegarder son statut professionnel. Pour ma part, je n'y suis jamais parvenu, sinon par un retour au mode logico-scientifique - comme le nomme Jérôme Bruner, cet autre avocat moderne du dualisme ${ }^{7}$ - dont le programme logiciste n'est à l'évidence qu'un sous-produit. Bref, le souhait d'un relais narratif, au-delà des limites de la construction savante, n'a rien à voir avec le désir d'affranchir celle-ci de toute référence ou révérence scientifique.

[7] Une autre manière de formuler le rejet du mode logico-scientifique, tenu pour inapplicable au monde de l'humain, consiste à invoquer une troisième voie de la connaissance, diversement située: entre science et littérature selon les uns, entre science et sens commun selon d'autres. La définition de cette voie moyenne revêt le plus souvent des formes négatives : chercheurs que nous sommes, nos constructions ne sauraient être assimilées à celles des littérateurs, journalistes ou autres narrateurs - 
trop libres - mais elles ne sauraient l'être non plus à celles de nos collègues des sciences naturelles ou des formalisations complètes - trop contraintes. Dès lors, comment les caractériser de façon positive, autonome, sans référence à ces deux pôles ? Ici encore, je n'y suis jamais parvenu; et il ne faut pas chercher ailleurs les sources de mon scepticisme à l'égard de la Troisième voie et de son avenir.

13 ET POURTANT... et pourtant, me dira-t-on, c'est bien elle qui l'emporte aujourd'hui, ô combien massivement, comme en témoignent non seulement les nombreux manifestes en sa faveur mais aussi et surtout l'écrasante prédominance des textes de sciences humaines qui ne "ressemblent ", en effet, ni à ceux des sciences naturelles, ni à ceux de la Littérature avec un grand L, ni à ceux de la prose commune. La suite de cet essai sera consacrée aux raisons qui me rendent insensible à cette objection de fait.

\section{Différends présents}

14 J'introduirai cette seconde partie en revenant à mon sujet véritable : la pérennité du questionnement logiciste. Cette affirmation doit surprendre tant les traces du phénomène sont minces, pour ne pas dire totalement absentes dans la littérature des sciences humaines. Je l'étaye néanmoins sur un certain nombre d'observations, déjà livrées ailleurs, que je me bornerai par conséquent à énumérer à la suite des précédentes, aussi laconiquement que possible.

15 [8] Commençons par déblayer le terrain des critiques adressées au programme logiciste. J'ai montré par ailleurs ${ }^{8}$ qu'elles reposaient pour la plupart sur des malentendus. L'erreur majeure porte sur les ambitions scientistes qu'on prête à ce programme, en oubliant qu'un de ses objectifs, affirmé dès l'origine et maintes fois rappelé, est de rendre manifeste la nécessité de ce que j'ai appelé plus haut un relais littéraire, au-delà des limites vite atteintes de la construction scientifique (supra, §5). Encore faut-il s'entendre sur cette expression: qu'est-ce qu'une construction "scientifique» appliquée aux œuvres ou aux conduites humaines? En adoptant les critères auxquels les sciences de la nature nous ont à cet égard habitués depuis quelques siècles, nous serions coupables, dit-on, d'ignorer l'immense littérature consacrée à la démonstration de leur irrecevabilité dans le domaine de l'humain. Nenni, ce n'est point ignorance, mais bien résistance, fondée sur le constat banal de la prédominance des critères en question, à long terme, dans l'édification de nos savoirs spécialisés.

16 [9] Où diable vais-je chercher de tels " constats »? Eh mais, dans ma propre discipline, pour commencer : l'archéologie, science historique s'il en fut, anthropologique aussi bien, puisqu'elle s'efforce de caractériser les sociétés anciennes sur les mêmes plans que l'ethnologie ou la sociologie face à des communautés vivantes. Mon expérience personnelle du «terrain» est limitée, j'en conviens; elle a suffi pourtant à me persuader de l'efficacité d'un savoir bâti dans son langage et dans sa logique propres (ou sales, si l'on veut : infra, \$12), lorsque, confrontés à des observations inédites, nous l'utilisons pour formuler des diagnostics, des prédictions, bref des interprétations capables de résister par la suite à l'épreuve de faits nouveaux, au moins pour un temps.

17 Curieusement, l'histoire des courants qui ont tour à tour occupé l'avant-scène en archéologie au cours des dernières décennies illustre bien cette suprématie têtue de l'ordre logico-empirique en matière de raisonnements interprétatifs, en dépit des entorses que les modes successives lui font subir. (A) Vint d'abord, dans les années 
cinquante, un mouvement opposé au laxisme intellectuel dont l'archéologie dite traditionnelle était jugée coupable: ce fut la "nouvelle archéologie", avec ses plaidoyers en faveur des pratiques scientifiques pures et dures - la mesure instrumentale, le quantitatif, substitués à l'impressionnisme de nos sens; la méthode hypothético-déductive, réputée plus rigoureuse que l'alliance traditionnelle de l'induction et de la confrontation empirique, etc. (B) Les critiques ne tardèrent pas, les unes soulignant la persistance des démarches « anciennes » et leur fécondité chez ceuxlà même qui les fustigeaient, les autres dénonçant l'anachronisme d'une apologie de la science dans le monde présent, agité par des mouvements inverses - postmodernisme, herméneutique, relativisme culturel, etc. (C) La mode suivante, dans les années quatrevingt, fut celle des mouvements en question: on y affirmait l'inanité du paradigme scientifique en archéologie, les biais inévitables de nos lectures du passé, certains condamnés (l'impérialisme occidental, rationnel, colonial, etc.), d'autres bienvenus (féminisme, tiers-mondisme, popularisme, etc.), et, last but not least (car nous sommes dans un contexte largement anglophone), la base sociale de la validation au lieu des références désuètes à l'empirisme. (D) Les libertés ainsi accordées à l'interprète eurent en archéologie le même effet qu'ailleurs : une abondante production de constructions dont Colin Renfrew, seigneur de l'archéologie britannique, avouait récemment que rien ne les distinguait à ses yeux des « most fantastical assertions of the lunatic fringe about flying saucers, earth magie and corn circles ». Le moment est venu, poursuit-il, d'en revenir à « the more careful and often the more painstaking delineation of arguments which can proceed more through the construction of frameworks of inference than by interpretive leaps ${ }^{9}$ ». On ne saurait mieux dire ; "a new undertaking ", ajoute Renfrew - Well, pas vraiment : c'est à quoi le programme logiciste s'emploie depuis vingt ans.

L'archéologie constituerait-elle en ces matières un cas particulier? Je n'en ai pas le sentiment. Même les plus relativistes des historiens en reviennent au témoignage des "faits » lorsqu'il s'agit d'invalider les thèses négationnistes ou créationnistes, par exemple, par des voies qui sont ou devraient être celles de la science avant celles de la morale ou de la religion. Et ce que je sais des pratiques de mes amis ethnologues, linguistes ou musicologues sur le terrain (et au-delà) me laisse penser que le raisonnement scientifique le plus « standard " garde là aussi tous ses droits.

[10] Un raisonnement néanmoins conduit, disais-je plus haut, « dans son langage et selon sa logique propres ». Il règne à cet égard d'autres malentendus. Le langage, d'abord: les engouements qu'on nous prête pour les systèmes de représentation artificiels iraient, a-t-on écrit, jusqu'au projet de substituer au langage naturel des codes ou des langages protocolaires unifiés, pour une discipline tout entière. Cette visée normative était présente et l'est encore dans la conception des systèmes de recherche documentaire, inévitablement: car le principe tant vanté du «traitement de l'information en langage naturel » ne résiste pas plus aujourd'hui qu'hier à l'examen ${ }^{10}$. Elle n'apparaît en revanche nullement dans la schématisation logiciste des constructions savantes: les bases de données que reconstitue l'analyse sont formulées dans le langage de l'auteur - langue naturelle ou « spéciale » selon le point de vue qu'on en a - sans transformation de la part de l'analyste (hormis le cas d'ailleurs délicat d'une traduction).

20 L'intention normative montre le nez, toutefois, lorsqu'on se place dans la perspective de l'étude des controverses. La démarche logiciste consiste alors à schématiser l'architecture des constructions concurrentes, relatives à un même objet, puis à 
cumuler ces schématisations dans une base de connaissances unique illustrant différentes manières d'interpréter les mêmes faits (de Po vers Pn) ou diverses façons d'étayer les mêmes hypothèses (de Pn vers Po) ${ }^{11}$. Les problèmes linguistiques, dès lors, se posent immédiatement, dans la mesure où l'appréciation du «même» et du "différent » passe par la reconnaissance des phénomènes de multi-vocité habituels : synonymies, polysémies, hétérogénéité des catégories de la description, etc., dans l'acception bien entendu relative de ces termes, selon l'univers de discours visé. Mais qu'y a-t-il là de choquant ou même de nouveau? Toute discipline qui se respecte est condamnée à des exercices de ce genre, avec ou sans la pression logiciste ; celle-ci n'est en l'espèce qu'un adjuvant, de par l'éclairage qu'elle projette sur les aspects sémiologiques des controverses et l'incitation forte qu'elle offre à les creuser.

21 [11] Après le langage, la logique de l'argumentation : le malentendu est ici du même ordre. L'analyse logiciste n'est pas une tentative de refonte du discours savant dans les moules de telle ou telle logique formelle. Elle repère et respecte les enchaînements que pratiquent les auteurs des textes visés, dans la syntaxe qui est la leur. La schématisation vise seulement à isoler les opérations d'inférence ou de réécriture auxquelles se ramènent ces enchaînements, débarrassés de leur expression rhétorique. Opérations de logique "naturelle ", déclarent certains, prompts à transférer au raisonnement le qualificatif qu'ils appliquent au langage des sciences de l'homme ${ }^{12}$. La réduction de ces opérations à des formules de réécriture $p \rightarrow q$ suggère néanmoins une lecture différente, tant nombre d'entre elles se révèlent éloignées du genre d'inférences pratiquées dans le discours ordinaire. Nos opérations logiques ne relèvent donc pas toutes $d u$ "raisonnement naturel ${ }^{13} »$ ou du sens commun; une partie d'entre elles constituent ce que j'ai proposé d'appeler, à la suite de Stephen Toulmin, des «logiques de champ ", aucunement formelles, mais qui n'en déterminent pas moins les cadres inférentiels où nos constructions savantes prennent forme, encouragées de temps à autre par quelques succès plus empiriques que mondains (infra, § 12).

S'agissant de l'étude des controverses, l'analyse logiciste a par ailleurs l'avantage de dégager les points précis où les divergences se produisent, d'une construction à l'autre, exprimées par des formules multivoques $\mathrm{p} \rightarrow \mathrm{q} 1, \mathrm{q} 2, \ldots \mathrm{qn}$... Je répéterai à ce propos ce que j'écrivais plus haut de l'apport sémiologique des schématisations: rien de bien neuf, toute discipline qui se respecte devant relever d'une manière ou d'une autre ce genre de défis intellectuels, mais adjuvant notable, quand même, de par l'inventaire systématique des bifurcations du raisonnement constatées dans un univers de discours donné.

Les logiques de champ vers lesquelles on tend de la sorte n'ont pas l'universalité de la logique; elles se constituent sur un tout autre plan, par la seule énumération des règles de dérivation (inférences, déductions) jugées recevables dans un domaine de recherche précis. Ne devrions-nous pas dès lors abandonner toute référence à la logique et nous contenter d'évoquer des bases de règles, comme en intelligence artificielle? Dans l'état primitif des recherches en la matière, je n'y verrais pour ma part aucun inconvénient.

[12] Ainsi, sur le plan du langage et de la logique, le programme logiciste n'a pas les hautes ambitions dont on lui fait parfois grief. Rien n'interdirait même d'en rester à son aspect descriptif, en résistant aux attraits normatifs de l'entreprise. Considérons par exemple un sujet d'étude labouré par plusieurs chercheurs - le suicide, les femmes en politique, l'émergence de l'État, la pédophilie - et sur lequel ceux-ci entretiennent des vues différentes, matière à controverses; l'analyse logiciste de leurs constructions 
respectives fournira l'inventaire des lieux où ces constructions divergent. Les uns se situeront dans le choix et dans la formulation des données (données observées, sans doute, mais aussi données présupposées, tirées de savoirs tenus pour établis ou des croyances de l'auteur), les autres dans la nature des opérations de dérivation ou de " détachement ", comme les nomme Marie-Jeanne Borel ${ }^{14}$, qui relient ces données aux hypothèses/conclusions avancées par chacun. Dans les deux cas, le but n'est pas de révéler le caractère "sale» des outils employés, linguistiques ou logiques: nous le connaissions ou le pressentions sans la (dé)monstration logiciste ; et celle-ci n'implique en outre aucun jugement quant à la valeur des théories ainsi bâties, de quelque façon qu'on choisisse de l'éprouver.

Et après ? L'analyste quant à lui, peut s'arrêter là. Mais s'il est aussi chercheur et s'il a quelque curiosité ou compétence en la matière, il serait surprenant qu'il ne fût pas tenté d'aller plus loin, par une analyse désormais critique et non plus seulement descriptive des cheminements concurrents - les siens compris, bien entendu, s'il a eu la sagesse de se compter au nombre des auteurs visés. Dans ce dernier cas, qui est le meilleur des cas $^{15}$, l'objectif ne sera pas d'«expliquer» les divergences par les biais personnels ou les contextes socio-historiques de chacun (supra, §1), mais bien de s'employer à les «résoudre", fût-ce à long terme, par un double mouvement d'enrichissement et d'épuration des schématisations - enrichissement lorsqu'on s'attache à préciser le domaine de validité de telle ou telle des constructions concurrentes ; épuration lorsque le jeu des contre-exemples conduit à écarter telle ou telle autre, mise en défaut sur l'un et/ou l'autre des deux axes de la schématisation (l'axe horizontal des données $\{\mathrm{Po}\}$, en extension, et l'axe vertical des opérations de réécriture $\{\mathrm{Pi}\} \rightarrow\{\mathrm{Pi} \pm 1\}$, en compréhension).

[13] Ce double mouvement n'est autre, on l'a compris, que celui de la réfutation des conjectures, dans le langage poppérien; et il n'est praticable qu'à deux conditions. La première, "objective", est que le champ des observations empiriques accessibles autorise le jeu des infirmations/confirmations; l'autre, "subjective", est qu'on ait envie de jouer à ce jeu. Si la première condition n'est pas remplie, il me semble assez clair, avec ou sans Popper, que nous ne sommes plus dans un univers de discours scientifique au sens usuel du terme, sauf à lui trouver une autre signification de nature à sauvegarder l'expertise, la profession ou le statut de l'interprète. C'est à quoi d'excellents esprits s'emploient inlassablement depuis des années. Depuis des années aussi, je rabâche mon inaptitude à donner une forme opératoire à leurs propositions (supra, § 7), sans pour autant méconnaître les limites du modèle scientifique - avec ou sans Popper - pour éclairer les ressorts des affaires humaines (supra, §5). Voilà bien une controverse, par conséquent, digne de figurer parmi celles dont il sera question dans cette revue. Son intérêt est le niveau où elle se situe : peut-on aborder l'analyse des controverses engagées sur un sujet quelconque, dans le cadre d'une "science " humaine donnée (histoire, sociologie, etc.), lorsqu'on entretient des vues différentes sur le genre de démarches intellectuelles que recouvre le terme entre guillemets?

Des vues différentes mais non point inconciliables, objectent certains, puisque nous semblons nous accorder au moins sur le caractère inévitablement hybride des constructions savantes, faites de morceaux apparentés à chacun des trois genres de discours dont il a été question plus haut (scientifique/logique, naturel/ordinaire, narratif/littéraire). Sans doute; mais les constructions des sciences naturelles font elles-mêmes appel à ces trois genres, à l'occasion. La différence est qu'elles ne les 
mélangent pas, ou pas au même degré que nos écrits habituels dans les sciences de l'homme. J'ai résumé ailleurs ce contraste par une formule assez simple: les produits d'un raisonnement conduit successivement sur le mode $\mathrm{A}$, puis sur le mode $\mathrm{B}$, ne sont pas les mêmes que ceux d'un raisonnement où les deux modes sont fondus en un troisième, $\mathrm{C}^{16}$.

Cette controverse épistémologique sera-t-elle un jour tranchée ? Probablement, au fil des âges : je veux dire par là que je n'imagine pas qu'elle puisse l'être autrement que par l'évolution des sciences humaines elles-mêmes, à une échelle de temps plus longue qu'on ne la considère généralement dans des débats de ce genre. Une chose me parait sûre en tout cas : c'est qu'on ne saurait guère attendre de la philosophie qu'elle ajoute quelque idée neuve en la matière, après toutes celles dont ses innombrables productions sur le sujet sont remplies. Plutôt qu'à un débat d'idées, il faut s'attendre selon moi à un processus de sélection plus ou moins darwinien, étalé sur plusieurs générations, où certains types de constructions savantes céderont progressivement la place à d'autres, pour des raisons qui tiendront largement aux vertus ou aux vices épistémologiques de chacune. Je terminerai cet essai par un rappel des observations qui fondent ce pari, plus abondamment exposées dans d'autres écrits.

\section{Prospective}

[14] Je citerai d'abord, sous un titre non provocateur, les progrès sensibles de la falsification (au sens français du terme). Ce n'est pas que la population des faussaires soit en croissance par rapport à la démographie générale, ni que ses techniques soient aujourd'hui plus perfectionnées qu'hier : mon ignorance est totale sur ces deux points. Je suis frappé en revanche par le nombre élevé des études consacrées de nos jours à la falsification dans les sciences humaines, comme aussi par le goût que nombre de chercheurs manifestent pour la production de pastiches dans leurs spécialités respectives. Les deux phénomènes sont sans doute liés, mais il est préférable de les considérer séparément.

Que les sciences humaines s'attachent de plus près que jadis à la falsification n'est que justice : les sciences naturelles n'ont jamais eu cette vocation puisque, par définition, la Nature ne produit pas de faux... Reste, bien sûr, la falsification des expériences ou des observations dont les sciences en question ont toujours été à l'affût, bien avant que les sociologues de la connaissance ne les prennent pour cible; mais ce n'est pas de ce phénomène que je veux parler. Les falsifications les plus intéressantes pour les sciences de l'homme sont celles qui portent sur leurs propres objets d'étude: les textes littéraires, par exemple, écrits "à la manière de » tel ou tel auteur, ou encore les monuments ou les sites archéologiques fabriqués de toutes pièces, les tableaux, sculptures ou autres œuvres écoulées sur le marché de l'art sous des paternités fictives, etc. Les faussaires de cet ordre ont le plus souvent des motivations ludiques ou commerciales plutôt que didactiques; leurs productions n'en soulèvent pas moins des questions intellectuelles intéressantes lorsque les spécialistes livrent, à propos de telles œuvres, des commentaires savants qui les authentifient, mais qu'on oublie promptement dès que la contrefaçon est dévoilée. Je ne crois pas nécessaire de préciser la nature de ces questions, sinon pour rappeler qu'elles prennent parfois un tour piquant lorsque la critique savante dénonce des faussaires qui n'en sont pas, jusqu'à les traduire en justice pour un délit qu'ils n'ont pas commis (je pense par exemple à ces 
étudiants d'un atelier de sculpture à Bologne, coupables d'avoir jeté dans un ruisseau, sans malice, des plâtres où ils s'étaient essayés à la manière de Modigliani; la découverte fortuite de ces œuvres fut accueillie avec enthousiasme par des experts, qui n'hésitèrent pas à poursuivre les étudiants pour faux témoignage lorsque ceux-ci, croyant bien faire, racontèrent l'histoire vraie des plâtres).

31 Une objection commune est que ces méprises sont somme toute assez rares et que la science finit toujours par triompher. La seconde proposition s'appuie sur des cas tout à fait réels où le recours à des techniques issues des sciences naturelles lève les incertitudes que les diagnostics divergents des experts laissaient subsister. Mais c'est mal raisonner : on change ici de registre, en laissant intacts des problèmes théoriques que ces divergences n'en continuent pas moins à poser, dans l'univers de discours qui est le leur. Bien plus, en s'adressant à des catégories d'analyse étrangères à cet univers, on semble admettre les limites de celles qui lui sont propres, plus langagières qu'instrumentales. Il n'est dès lors pas surprenant que la lente accumulation des cas de ce genre au fil des ans, dans toute espèce de domaines de recherche (littérature, archéologie, histoire de l'art, ethnologie, etc.), finisse par engendrer un questionnement qui dépasse les récits anecdotiques de falsification.

Est-ce parce qu'ils pressentent la prégnance du sujet que nombre de chercheurs sont aujourd'hui portés à se falsifier eux-mêmes, en quelque sorte, en inventant de "faux » objets d'étude dont ils publient des analyses savantes, à la manière de celles qui portent sur les «vrais»? J'ai cité ailleurs quelques exemples du genre pour souligner la signification qu'ils revêtent dans la perspective logiciste. En prenant ses cheminements interprétatifs pour objet de pastiche, le chercheur les élève, fût-ce implicitement, à la dignité d'objets d'étude virtuels ; les travaux sur la simulation des raisonnements, dans le programme logiciste, ne sont, disais-je, que «la version explicite et matérielle du même projet ${ }^{17}$ ".

[15] La toute récente « affaire Sokal » offre une transition commode vers une deuxième famille d'observations touchant l'évolution probable des constructions savantes, à long terme. On connaît l'histoire : un professeur de physique à l'Université de New York, Alain Sokal, étonné des idées qu'entretiennent un grand nombre de philosophes, sociologues et autres spécialistes de l'ordre humain à propos des sciences naturelles, décide de tenter un pari. Est-il possible de faire paraitre dans une revue réputée de sciences sociales un article apparemment voué à la défense des mêmes idées, truffé de propositions absurdes sur le monde de la physique ou des mathématiques, analogues à celles qui étonnent Sokal, mais authentifiées en quelque sorte par la réputation professionnelle du physicien ? Le pari fut « hélas gagné », comme l'a raconté Sokal ; son article, au titre pourtant suspect («Transgressing the Boundaries: Toward a Transformative Hermeneutics of Quantum Gravity»), fut publié dans Social Text - «a leading North American journal of cultural studies whose editorial includes such luminaries as Frédéric Jameson and Andrew Ross ${ }^{18}$ ». La communauté savante fut piquée, à en juger par l'ampleur et la vivacité de ses réactions, d'abord aux États-Unis, puis, plus sourdement, en Europe quelques mois plus tard (voir le site «Sokal » sur Internet). Les arguments invoqués " pour » ou "contre » le pari de Sokal et les leçons qu'il faut en tirer mériteraient une exégèse au terme de laquelle, si j'en étais l'auteur, les sciences humaines ne sortiraient pas grandies. Pour mon présent propos, je retiendrai seulement de ces débats deux traits: d'une part, la répugnance de mes collègues à débattre des problèmes techniques que posent la contrefaçon de Sokal et 
son succès, sans y mêler toutes sortes de considérations socio-politiques pour la plupart malvenues, pour qui connaît l'auteur, et en tout cas sans rapport avec le sujet ; d'autre part, l'aimable hilarité que déclenchent les textes cités par Sokal à l'appui de l'Herméneutique Transformationnelle de la Gravité Quantique, qu'il s'agisse des thèses "fausses" qu'il s'attribue ou des pensées "vraies» de ses sources - philosophes, sociologues, psychanalystes - indifféremment. "Rions, mais jaune", écrivait Régis Debray au sujet de l'affaire, récupérée pour répondre aux fulminations de Pierre Bourdieu contre la médiatologie ${ }^{19}$; non pas, rions franchement, de bon cœur, mais aussi bien du vrai que du faux, indifféremment, et pour les mêmes raisons.

C'est de ce rire ou sourire que je voulais faire état au nombre des raisons de mon optimisme quant à l'avenir de nos constructions. Je l'aperçois en effet de plus en plus clairement sur les bancs des séminaires où sont décortiqués nos écrits - les miens compris - lorsque l'analyse met en évidence non pas les bouffonneries métaphoriques dont s'étonnent Sokal et ses pairs, mais, plus simplement, la relative banalité de nos argumentations et de leurs conclusions une fois débarrassées de leur emballage rhétorique. Le rire n'est alors ni jaune ni méchant, mais plutôt philosophique, au sens où l'entendait Bergson: il exprime seulement la surprise, et peut-être un certain soulagement, face à un phénomène soudain perçu comme saugrenu alors qu'on croyait jusque-là devoir s'en accommoder, sans joie. Je veux parler de la disproportion entre le volume de nos écrits et la dimension des idées neuves qu'ils véhiculent, une fois réduits à leurs composantes essentielles (supra, § 1). Entendons-nous bien, cependant : les bases de «faits" recueillis peuvent être immenses (avec toutes les réserves d'usage sur l'emploi de ce terme), comme dans ces grands et beaux ouvrages d'érudition où se mesure d'abord le progrès des connaissances historiques, linguistiques et autres ; mais les bases de règles, pour m'en tenir au jargon logiciste (supra, §11), n'ont pas ces dimensions, ni par conséquent les théories qu'elles engendrent, en dépit des longueurs de la prose où nous avons l'habitude de les coucher. Les vertus éventuelles de celle-ci sur le plan littéraire, toutes souhaitables qu'elles soient, ne changent rien à ce constat.

Revenons maintenant à notre sujet. Il est clair que, vus sur la toile de fond qui précède, nos conflits d'interprétation risquent de paraitre diminués. Ce n'est pas que l'analyse logiciste des constructions concurrentes réduise leurs divergences à peu de chose, mais plutôt qu'elle tend à désamorcer les controverses engagées à leur sujet. Le côté ludique des exercices de contrefaçon déteint en quelque sorte sur les exercices de l'interprétation savante, où l'on en vient à flairer sinon des jeux de société, du moins une activité cousine de l'explication de texte ou de la dissertation scolaire, sans plus de gravité. Reste, bien sûr, le côté sérieux de l'affaire que lui confèrent les sanctions institutionnelles; les milliers de mémoires soumis chaque année à l'autorité universitaire sur les sujets les plus variés en sont pour ceux qui les écrivent la manifestation la plus palpable. Le fait nouveau, tel du moins que je le perçois à travers l'enseignement, est une impatience grandissante chez les étudiants et chercheurs jeunes astreints à ces exercices, fondée sur la conscience d'un certain anachronisme des modes de discours et de pensée pratiqués, les premiers déterminant largement les seconds. Le malaise ne disparaît pas forcément avec l'obtention des diplômes; il est entretenu par le flot des interprétations expressément bâties « à la manière de » tel ou tel paradigme - le structuralisme, les Trois fonctions, le Carré sémiotique, les modèles dynamiques, etc. - mais qu'il convient d'accueillir désormais sans les connotations qui s'attachent à la locution entre guillemets. Passé un certain niveau de réflexion épistémologique ou sociologique, la conversion n'est pas toujours facile; elle peut 
même en décourager quelques-uns lorsqu'il s'agit non plus de consommer mais de produire des textes de la même facture, sauf à se contenter des critères de validité consensuelle honorés dans la mouvance herméneutique ou postmoderne.

Tous ne s'y résignent pas ; mais que faire dans ce cas? La question m'a été posée plus d'une fois, comme elle l'a sans doute été à d'autres, par ces mêmes étudiants et chercheurs jeunes que je viens d'évoquer. Ma réponse a toujours été la même: «le double jeu », par quoi j'entends d'une part le respect des pratiques discursives établies, à des fins évidentes et parfaitement justifiées, d'autre part la recherche discrète de formes d'écriture et d'argumentation plus serrées, au service de plaisirs personnels d'un autre ordre. Est-ce trop demander? Je ne crois pas: rappelons-nous que ce dédoublement n'est pas le premier du genre. Nos devanciers dans les sciences naturelles, par exemple, ont dû jadis passer par là lorsque les langages et les raisonnements de la "nouvelle science " prenaient corps, après Bacon et Galilée, tandis que les traditions de la philosophie naturelle se maintenaient dans les universités. Mais il y a plus : si l'accélération de l'histoire n'est pas une vaine formule, on peut imaginer que l'évolution logico-linguistique dans les sciences de l'homme prendra pour sa part moins de temps. Ma troisième et dernière famille d'observations porte sur quelques signes en faveur de ce pari.

[16] Je les rassemblerai sous un seul titre : la crise de l'information scientifique et des publications savantes dans nos disciplines. L'expression couvre plusieurs thèmes différents. Le plus commun est d'ordre financier : nos publications coûtent en général plus d'argent qu'elles n'en rapportent, l'État est donc appelé à remplacer ou à subventionner les éditeurs privés, mais les crédits publics ne suffisent plus aujourd'hui à garantir l'impression de tous les livres et revues que produisent les sciences de l'homme. Les solutions proposées mettent toutes en cause, d'une manière ou d'une autre, nos pratiques discursives présentes, et c'est à ce titre qu'elles intéressent mon propos. On nous suggère d'écrire " autrement " pour un public plus large (où l'on en revient aux genres journalistiques ou littéraires), ou d'écrire "moins", selon des stratégies de recherche plus réfléchies (où l'on retombe inévitablement sur les problèmes d'évaluation qui occupent dans le questionnement logiciste une si grande place).

38 Vient ensuite l'aspect technologique de la crise : on attend des nouvelles technologies de l'information qu'elles la dénouent, grâce au transfert d'une partie de nos connaissances scientifiques vers les réseaux d'ordinateurs, au lieu des publications imprimées. Se posent alors les problèmes de langage et de logique soulevés dans la perspective logiciste (supra, § 10 et $\S 11$ ).

39 La dimension la plus profonde de la crise de l'information est néanmoins tout autre, ni technique ni financière : elle tient au déséquilibre que nous connaissons tous mais dont nous parlons peu entre le volume de nos productions écrites et les limites de nos capacités de lecture. Il en résulte des situations paradoxales, où nous rédigeons des travaux savants comme s'ils devaient être lus, alors que nous savons à l'avance qu'ils ne le seront guère, à proprement parler. La raison la plus favorable de ce pronostic tient au plafonnement inévitable de nos temps de lecture, face aux dépassements que l'abondance des publications "pertinentes" nous impose dans quelque domaine de recherche que ce soit. Je me suis souvent fait l'écho des débats engagés dans d'autres pays sur ce problème, en Grande-Bretagne notamment ; il faudra bien qu'un jour nous les engagions, de ce côté-ci de la Manche. La schématisation des constructions savantes, 
dans le programme logiciste, ne prétend nullement apporter la solution; elle a seulement le mérite de prendre en compte deux recommandations régulièrement avancées dans les débats en question, touchant l'une la condensation souhaitable de nos écrits, l'autre la recherche de formes de présentation propices à la consultation plutôt qu'à la lecture, celle-ci étant réservée à des textes d'un autre ordre où la littérature reprend tous ses droits.

Ainsi, la crise de l'information entraîne pour des raisons pratiques le genre de réflexion que propose l'analyse logiciste à des fins théoriques. On m'objectera que l'immense majorité des chercheurs rejette tout renoncement aux formes d'écriture traditionnelles et «naturelles » cultivées dans les sciences de l'homme depuis des lustres. C'est tout à fait vrai, et je ressens autant que d'autres le poids des contraintes intellectuelles qu'imposent les schématisations ; mais toute manière meilleure de relever à l'avenir le défi s'accompagnera vraisemblablement des mêmes contraintes. En outre, nos résistances sont de celles qu'on attend à l'approche de tout changement dans l'ordre établi des choses ; il serait imprudent par conséquent de se recommander d'elles seules pour vouer les essais logicistes aux gémonies. Enfin, les minorités silencieuses méritent dans les temps de mutation autant d'attention que les voix majoritaires; or, il en existe déjà quelques-unes, discrètement engagées dans des travaux sur la rhétorique des textes de sciences humaines à des fins qui comprennent leur refonte éventuelle, à long terme, dans une perspective technique et non philosophique assez proche de la nôtre ${ }^{20}$.

41 Je terminerai par où j'ai commencé : ma gêne à redire, à travers les 16 points qui précèdent, des idées que j'ai déjà présentées ailleurs dans des contextes variés. Ma première excuse est la croyance tenace des éditeurs de la présente revue en l'utilité d'un exposé de plus, en rapport avec le thème des controverses. Ce rapport n'est pas immédiat; il s'est même obscurci sans doute à mesure que j'avançais, jusqu'à traiter d'affaires telles que la forme des publications savantes. Mais tout se tient: nos traditions d'écriture, nos façons de raisonner et, dans ces raisonnements, nos libertés d'interprétation, génératrices de controverses. L'analyse logiciste de l'ensemble soulève des questions de tous ordres dont on devrait trouver quelques traces dans les articles qui suivent, fût-ce indirectement. Ma seconde excuse, quant aux redites, est qu'en les plaçant dans le présent recueil, je donne à chacun comme à moi-même l'occasion de mesurer l'à-propos du titre que j'avais d'abord donné à ce texte: la pérennité du questionnement logiciste.

\section{NOTES}

1. Sont rangés sous ce titre un ensemble de travaux conduits depuis les années soixante-dix dans divers secteurs des sciences de l'homme, mais plus particulièrement en archéologie, dans les perspectives d'une épistémologie pratique. Sur les objectifs et la démarche générale, cf. J.C. Gardin, "Vers une épistémologie pratique en sciences humaines ", in J.-C. Gardin, M.-S. Lagrange, J.-M. Martin, J. Molino, J. Natali-Smit, La logique du plausible. Essais d'épistémologie pratique en sciences humaines, Paris, Éd. de la MSH, 1981, p. 3-91 (2 éd., 1987, p. 27-100) ; J.C. Gardin, «Questions d'épistémologie pratique dans les perspectives de l'intelligence 
artificielle ", Bulletin de la Société française de Philosophie, 81-3, 1987, p. 60-112, reproduit dans J.C. Gardin, Le calcul et la raison. Essais sur la formalisation du discours savant, Paris, Éd. de l'EHESS, 1991, p. 59-89. La parenté entre le programme logiciste et l'école philosophique du même nom est lointaine, mais elle a paru suffisante pour justifier l'étiquette ; cf. sur ce point J.-C. Gardin, Une archéologie théorique, Paris, Hachette, 1979, p. 24-38. Pour un aperçu des travaux apparentés au programme logiciste, cf. J.-C. Gardin, «Formalisation et simulation des raisonnements ", in J. Revel et N. Wachtel, eds, Une école pour les sciences sociales. De la VI section à l'École des Hautes Études en Sciences Sociales, Paris, Cerf/Éd. de l'EHESS, 1996, p.185-308; J.-C.Gardin et M. N. Borghetti, L'architettura dei testi storiografici : un'ipotesi, Bologne, CLUEB, 1995, p. 45-47.

2. La coexistence de points de vue ou de théories multiples sur un même sujet est un phénomène courant dans les sciences de la nature, chacun le sait; mais elle est considérée là comme une source de problèmes qu'il importera tôt ou tard de résoudre, non par des considérations d'ordre socio-historique mais par « les voies habituelles de la recherche dans les disciplines empiriques », selon ma formule. Je ne rappelle cette banalité que pour marquer la différence avec la "problématologie» du philosophe M. Meyer, par exemple, malgré l'apparente identité du propos: "How should we proceed", écrit-il, «when we are confronted with several possible, compatible interpretations? How can we rationally proceed if we agree to exclude intuition or revelation, to arrive at one interpretation and justify this choice, if we do not have some technique of understanding at our disposal?» (M. Meyer, Meaning and Reading. A Philosophical Essay on Language and Literature, Amsterdam, John Benjamin, 1983, p. 146). La problématologie serait la science ou la technique offerte par M. Meyer pour résoudre ces conflits d'interprétation, dans l'esprit des "théories générales de la signification " auxquelles l'auteur ne manque pas de se référer (C. Peirce, P. Ricœur, E. Betti et bien d'autres). Mais hélas, on ne trouve dans aucune de ces théories ou de ces voies (problématologie, sémiotique, herméneutique) rien qui ressemble aux « procédures rationnelles » ou aux « techniques interprétatives » annoncées; cf. sur ce point J.-C. Gardin, «Fondements possibles de la sémiologie », Recherches sémiotiques/Semiotic Inquiry, 5, 1985, p. 1-30, reproduit dans J.-C. Gardin, Le calcul et la raison, op. cit., p. 201-226.

3. La plupart des maîtres en explication de textes littéraires en sont, j'en suis sûr, tout à fait convaincus, même s'il est rare qu'ils prennent la peine d'en tirer explicitement toutes les conséquences comme l'a fait Élisabeth Ravoux Rallo (Méthodes de critique littéraire, Paris, Armand Colin, 1993).

4. J.-C. Gardin, Les analyses de discours, Neuchâtel, Delachaux et Niestlé, 1974, p. 56-60, reproduit dans Le calcul et la raison, op. cit., p. 123-126 ; Une archéologie théorique, op. cit., p. 295-300.

5. J.-C. Gardin, Le calcul et la raison, op. cit., p. 36-37.

6. J. Revel a raconté cette évolution dans «Ressources narratives et connaissance historique", Enquête, Anthropologie, Histoire, Sociologie, 1, Les terrains de l'enquête, 1995, p. 43-70.

7. J. Bruner, "Two Modes of Thought", in Actual Minds, Possible Worlds, Cambridge, Harvard University Press, 1986, p. 11-43.

8. J.-C. Gardin et M. N. Borghetti, L'architettura dei testi storiografici..., op. cit., p. 87-110.

9. C. Renfrew et E. Zubrow, eds, The Ancient Mind. Elements of Cognitive Archaeology, Cambridge, Cambridge University Press, 1994, p. 9 et 11.

10. Cf. à ce sujet J.-C. Gardin, "Informatique et progrès dans les sciences de l'homme ", Revue Informatique et Statistique dans les Sciences humaines, 30-1 à 4,1994, p. 15-18.

11. Exemples: M.-S. Lagrange et M. Renaud, «SUPERIKON, essai de cumul de six expertises en iconographie ", in J.-C.Gardin, O. Guillaume, P. Q. Herman, A. Hesnard, M.-S. Lagrange, M. Renaud, E. Zadora-Rio, Systèmes experts et sciences humaines. Le cas de l'archéologie, Paris, Eyrolles, 1987, p. 191-229 ; H.-P. Francfort, « The Sense of Measure in Archaeology : An Approach to the Analysis of Proto-Urban Societies with the Aid of an Expert System », in J.-C. Gardin et C. S. Peebles, eds, Representations in Archaeology, Bloomington-Indianapolis, Indiana University Press, 1992, p. 291-314. 
12. Cf. en particulier J.-B. Grize, "Logique mathématique, logique naturelle et modèles ", in Jahresbericht der Schweizeriscben Geisteswissenschaftlichen Gesellschaft, 1974 («Sciences humaines et formalisation »), p. 201-207 ; J.-B. Grize, ed., Logique naturelle du raisonnement, Travaux du Centre de Recherches Sémiologiques, 41, Université de Neuchâtel, 1982 ; citations plus récentes du même auteur in J.-C.Gardin, "Calcul, informatique et raisonnement en archéologie ", in D. Miéville, ed., Raisonnement et calcul, Actes du colloque de Neuchâtel des 24-25 juin 1994, Travaux du Centre de Recherches Sémiologiques, 63, Université de Neuchâtel, 1995, p. 17-21.

13. J.-C. Passeron, Le raisonnement sociologique. L'espace non-poppérien du raisonnement naturel, Paris, Nathan, 1991.

14. M.-J. Borel, in J.-B. Grize, ed., Raisonnements et raisons, Travaux du Centre de recherches Sémiologiques, 44, Université de Neuchâtel, 1983, p. a1.

15. Rappelons que le programme logiciste n'est pas une entreprise polémique, destinée à mettre en relief les failles des raisonnements de l'Autre. Nous avons très tôt voulu associer les auteurs eux-mêmes à l'analyse (ex. : M.-S. Lagrange et Ch. Bonnet, Les chemins de la Memoria. Nouvel essai d'analyse du discours archéologique, Paris, Éd. du CNRS, 1978), avant d'en venir à l'idée que le parti le plus sage, et en tout cas le plus paisible, était de prendre nos propres écrits pour objet (ex.: A. Gallay et C. Sauvain-Dugerdil, Le Sarnyéré Dogon. Archéologie d'un isolat, Mali, Paris, Éd. Recherche sur les Civilisations, 1981 ; J.-C. Gardin, «Les relations entre la Grèce et l'Asie centrale à l'époque hellénistique d'après les données céramologiques ", in J.-C. Gardin, O. Guillaume, etc., Systèmes experts et sciences humaines..., op. cit., p. 59-91; E. Zadora-Rio, "L'identification d'une construction médiévale », in ibid., p. 169-189 ; E. Evrard, « Horace, CI, 11 », Les Études classiques, 63, 1995, p. 23-37).

16. J.-C. Gardin, in J.-C. Gardin et M. N. Borghetti, L'architettura dei testi storiografici..., op. cit., p. $109-110$.

17. J.-C. Gardin, «La pensée réfléchie et ses progrès dans les sciences de l'homme ", § 2-4, à paraître in Le Genre humain (Actes du colloque de l'EHESS sur la «Contemporanéité en question », Toulouse, 7-8 avril 1995).

18. A. Sokal, «A Physicist Experiment with Cultural Studies", Lingua Franca, mai-juin 1996, p. 62-64, citation p. 62.

19. R. Debray, « Savants contre docteurs », Le Monde, 18 mars 1997.

20. Un exemple, parmi d'autres: un groupe d'enseignants italiens s'est formé autour d'Ivo Mattozzi, à l'Université de Bologne, pour repenser la pédagogie de l'histoire sur la base d'une analyse approfondie de la littérature spécialisée. Les points de rencontre avec le programme logiciste ne manquent pas, en particulier quant à l'évolution probable des modes de publication ou plus généralement de communication dans les disciplines historiques.

\section{RÉSUMÉS}

L'analyse logiciste des constructions savantes a pour but de mettre à nu leurs composantes symboliques : une base de données (observations et présuppositions) et un ensemble d'opérations de réécriture exprimant le raisonnement qui relie cette base aux thèses de la construction. Les travaux inspirés de ce programme depuis une vingtaine d'années soulèvent des questions intéressantes dans les perspectives d'une épistémologie pratique maintes fois exposées. L'étude des conflits d'interprétation y tient une large place ; elle s'apparente à l'analyse des controverses 
scientifiques mais vise moins à expliquer celles-ci, du point de vue socio-historique, qu'à mieux définir les voies choisies pour les résoudre, les éluder ou les dépasser, selon les cas. Le questionnement logiciste porte sur les conséquences intellectuelles et institutionnelles de ces choix. L'affirmation de sa pérennité procède à la fois d'un constat et d'un pari, l'un et l'autre argumentés dans cet article.

The logicist analysis of scholarly constructions aims at revealing their symbolic components: a data base (observations and presuppositions) and a whole lot of operations of rewriting expressing the reasoning which links this base to the thesis of construction. Works inspired from this programme since some twenty years raise interesting questions in the perspectives of a practical epistemology often exposed. The study of conflicts of interpretation holds a large place here: it is closely connected to the analysis of scientific controversies but aims less at explaining these from a socio-historical point of view, than at better defining the paths chosen to resolve them, elude them or go beyond them, according to the case. Logicist questioning is concerned with the intellectual and institutional consequences of these choices. The affirmation of its perpetuity follows from a statement and a bet at the same time, both argued in this article.

\section{AUTEUR}

\section{JEAN-CLAUDE GARDIN}

Jean-Claude Gardin (CNRS, EHESS) a partagé ses activités entre deux volets : l'archéologie de l'Asie centrale et l'analyse logiciste des constructions historiques. Ses ouvrages principaux sur le second volet sont : Les analyses de discours, Neuchâtel, Delachaux et Niesdé, 1974 ; Une archéologie théorique, traduction française, Paris, Hachette, 1979 (texte original anglais : Cambridge-Paris, Cambridge University Press-Editions de la Maison des Sciences de l'Homme, 1980); Le calcul et la raison, essais sur la formalisation du discours savant, Paris, Editions de l'EHESS, 1991 ; avec Maria Novella Borghetti, L'architettura dei testi storiografici : un'ipotesi, Bologne, CLUEB, 1995. 